\title{
Peripheral Embolization of Left Ventricular Thrombus Leading to Acute Bilateral Critical Limb Ischemia: A Rare Phenomenon
}

\author{
Khushboo K. Agarwal a, Steven Douedia , Abbas Alshamia, \\ Brook DeJene ${ }^{\mathrm{b}}$, Robert G. Kayser ${ }^{\mathrm{c}}$
}

\begin{abstract}
Left ventricular thrombus (LVT) is a well-known complication of myocardial infarction (MI) leading to significant morbidity and mortality. LVT can also lead to systemic thromboembolic events causing threatening limb ischemia. We report a rare case of critical bilateral limb ischemia that resulted from peripheral embolization of LVT post MI, which was managed successfully by emergent surgical intervention and anticoagulation. A 74-year-old male with a medical history of hypertension, diabetes, hyperlipidemia and coronary artery disease status post stenting of the left anterior descending and left circumflex arteries presented to the emergency department with typical chest pain and progressive shortness of breath. Cardiac troponin levels on admission were $35 \mathrm{ng} / \mathrm{mL}$ of blood. The patient subsequently underwent emergent cardiac catheterization which revealed significant triple vessel disease, and was referred for coronary artery bypass grafting (CABG) surgery. Transthoracic and transesophageal echocardiograms revealed the presence of an apical aneurysm with chronic organized mobile thrombus at the apex. Post CABG, the patient complained of excruciating right leg pain. Computed tomography (CT) angiogram of the abdominal aorta and lower extremities revealed a large embolus at the aortic bifurcation occluding the right and nearly occluding the left common iliac arteries and thrombus in the right popliteal artery. He underwent emergent vascular surgery with resolution of his symptoms and remained without further complications. The incidence of LVT remains high in post-MI patients, and complications of LVT are known to include thromboembolic events. Peripheral embolization of acute or chronic LVT leading to bilateral distal embolization and critical limb ischemia remains a rare occurrence. This case report aims to aid clinicians to recognize and
\end{abstract}

Manuscript submitted February 4, 2020, accepted February 14, 2020

aDepartment of Medicine, Jersey Shore University Medical Center, Neptune, NJ 07753, USA

${ }^{b}$ Department of Cardiothoracic Surgery, Jersey Shore University Medical Center, Neptune, NJ 07753, USA

'Department of Cardiology, Jersey Shore University Medical Center, Neptune, NJ 07753, USA

${ }^{\mathrm{d}}$ Corresponding Author: Steven Douedi, Department of Medicine, Jersey

Shore University Medical Center, Neptune, NJ 07753, USA.

Email: Steven.Douedi@hackensackmeridian.org

doi: https://doi.org/10.14740/cr1030 promptly manage LVT and related arterial thromboembolic events with anticoagulation and emergent surgical intervention if limb ischemia develops.

Keywords: Ischemia; Ventricular thrombus; Peripheral embolization; Myocardial infarction; Thromboembolism

\section{Introduction}

Left ventricular thrombus (LVT) is a well-known complication of left ventricular dysfunction, most commonly occurring post anterior ST-segment elevation myocardial infarction (STEMI) $[1,2]$. While the incidence of LVT post myocardial infarction (MI) has decreased over the years due to rapid percutaneous coronary intervention (PCI), it remains a significant cause of morbidity and mortality [3]. LVT is associated with systemic thromboembolism more frequently seen in non-ischemic causes $[2,4,5]$. Acute embolic occlusion of the bilateral lower extremities remains an extremely rare finding. This phenomenon is a medical emergency and requires emergent surgical intervention, which can lead to excellent patient outcomes [6]. We herein report a rare case of critical bilateral limb ischemia that resulted from peripheral embolization of LVT that was managed successfully by emergent surgical intervention.

\section{Case Report}

A 74-year-old male with a medical history of hypertension, diabetes, hyperlipidemia and coronary artery disease status post stenting of the left anterior descending and left circumflex arteries presented to the emergency department with typical chest pain and progressive shortness of breath. Physical assessment was unremarkable on admission. Laboratory investigation was normal except for hemoglobin A1c of $8.3 \%$ and a cardiac troponin level of $35 \mathrm{ng} / \mathrm{mL}$. The patient was loaded with $325 \mathrm{mg}$ of aspirin and $180 \mathrm{mg}$ of ticagrelor. The patient subsequently underwent emergent cardiac catheterization which revealed significant triple vessel disease, and was referred for coronary artery bypass grafting $(\mathrm{CABG})$ surgery. Preoperative workup included a carotid ultrasound which demonstrated no 

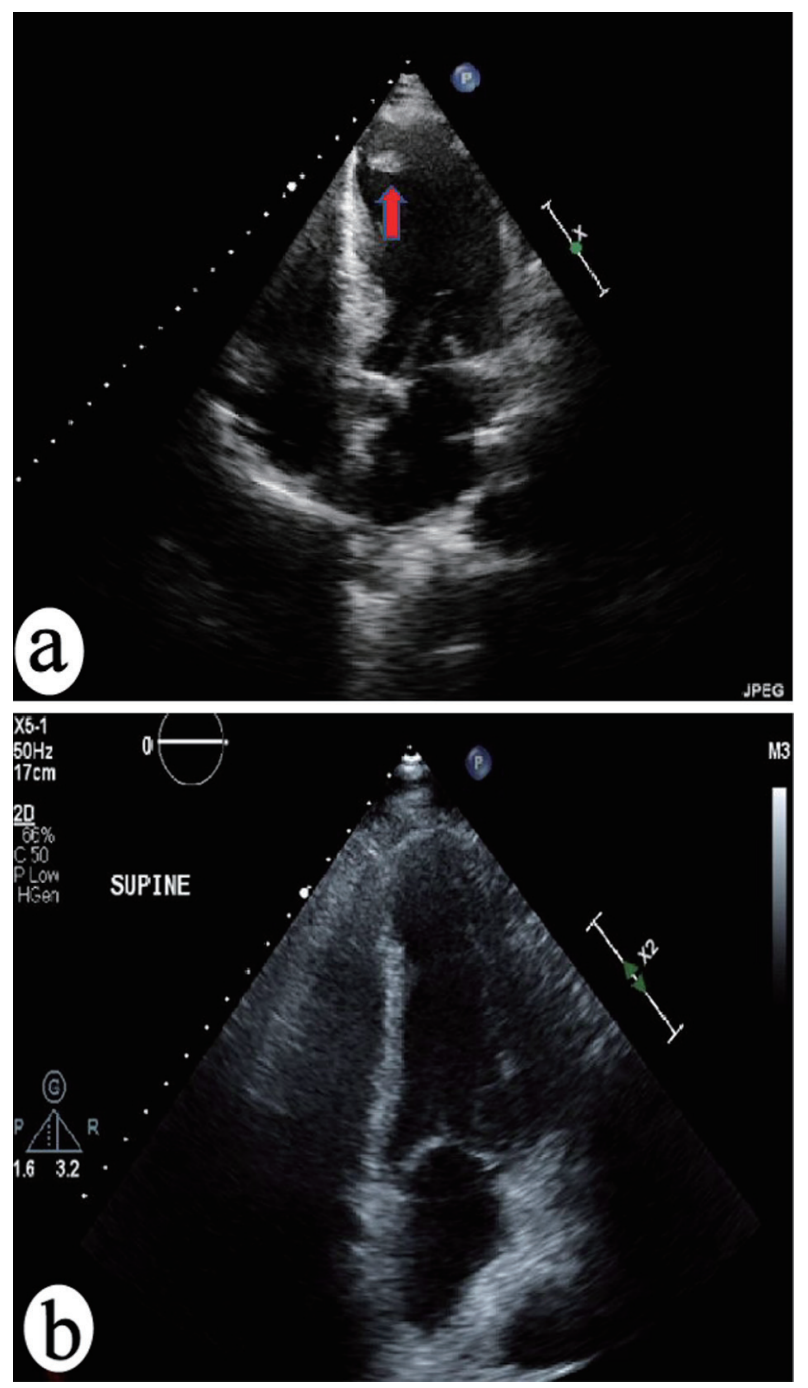

Figure 1. (a) Transthoracic echocardiogram (TTE) done pre-CABG surgery showing a mobile left ventricular (LV) apical thrombus associated with an LV aneurysm. (b) TTE done post-CABG surgery and anticoagulation treatment showing decreased size of LV apical thrombus.

significant stenosis and a transthoracic echocardiogram (TTE), which showed a mobile left ventricular (LV) apical thrombus associated with an LV aneurysm (Fig. 1). Transesophageal echocardiogram (TEE) was also performed and confirmed the presence of an apical aneurysm with chronic organized mobile thrombus at the apex. He was started on a continuous lowintensity heparin drip $(25,000$ units in $250 \mathrm{~mL} \mathrm{D} 5 \mathrm{~W})$ and 81 $\mathrm{mg}$ aspirin daily, and the heparin drip was held about $2 \mathrm{~h}$ prior to his procedure. He underwent a successful CABG procedure and remained hemodynamically stable. Shortly after awakening from anesthesia, the patient complained of excruciating right leg pain, and was found to have significant discoloration with diminished pulses in both lower extremities. Computed tomography (CT) scan of the abdomen and lower extremities revealed a large embolus at the aortic bifurcation occluding the right common iliac and nearly occluding the left common iliac artery and thrombus in the right popliteal artery (Fig. 2).
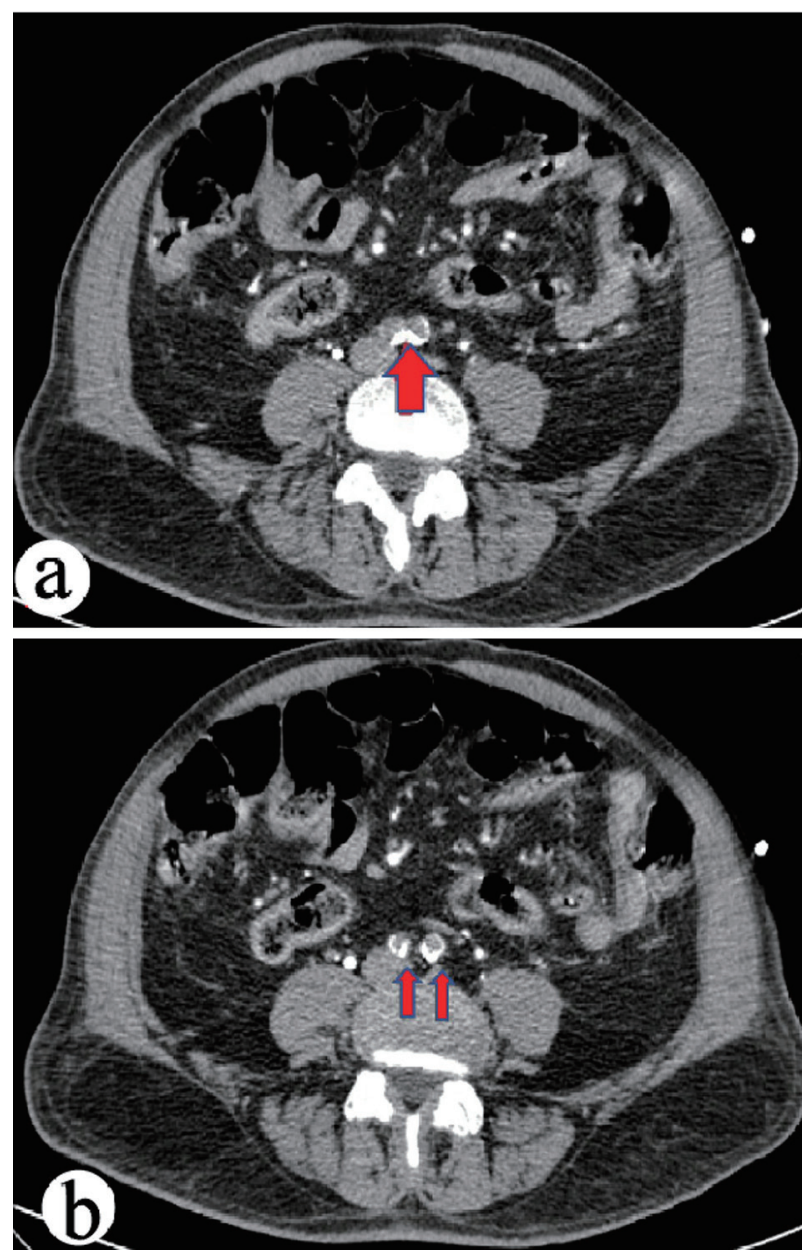

Figure 2. (a) Computed tomography (CT) scan of the abdomen showing a large embolus at the aortic bifurcation. (b) CT scan of the abdomen showing occlusion of the right common iliac and near occlusion of the left common iliac artery.

CT angiogram of the abdominal aorta was also performed confirming the findings (Fig. 3). He was taken emergently back to the operating room for an emergent right and left femoral cut down with repair of common femoral arteries, aortic right iliac artery and right popliteal artery thromboembolectomy. Postoperatively he was started on unfractionated heparin, oral clopidogrel and warfarin. Repeat echocardiogram revealed that the LV apical thrombus was still present but was noted to be smaller in size compared to the preoperative study (Fig. 1). His hospital course thereafter remained unremarkable and he was discharged with a normal physical exam and on warfarin, clopidogrel, and aspirin for anticoagulation.

\section{Discussion}

LVT formation is commonly seen after acute MI, such as in the patient presented in this case, and dilated cardiomyopathy [7]. The incidence of LVT is up to $15 \%$ in patients post MI, and even higher in patients with anterior MI [5]. The American 


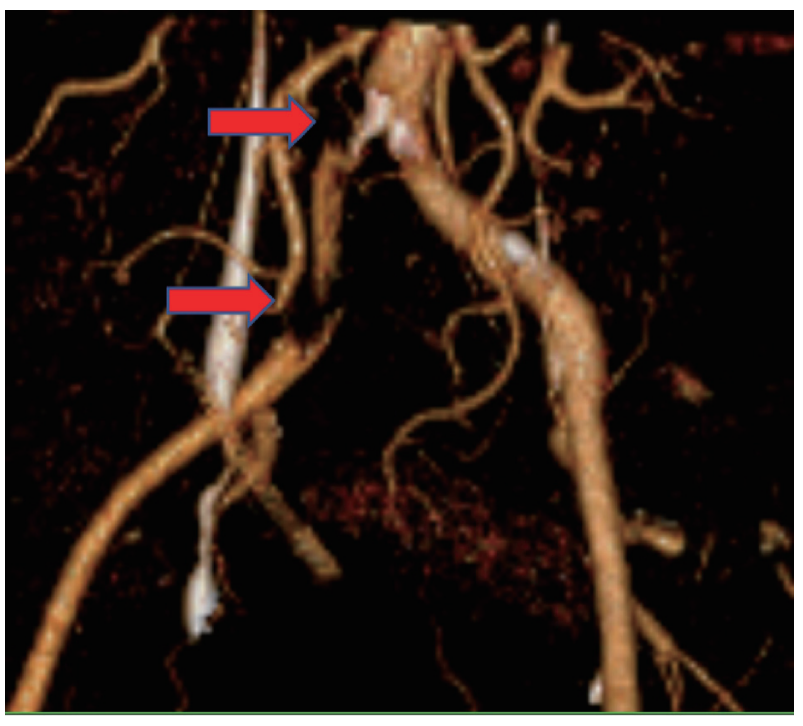

Figure 3. Computed tomography (CT) angiogram of the abdominal aorta and lower extremities showing right common iliac and right popliteal artery occlusion.

College of Cardiology (ACC) has presented TTE as the screening modality of choice in patients presumed to have LVT, as performed in our patient. The pathophysiology of LVT formation is similar to other causes of thrombi formation, and can be explained by Virchow's triad: hypercoagulable state, stasis, and endothelial injury in addition to a low ejection fraction seen after a MI [8]. LV apical thrombi have also been associated with systemic thromboembolic events such as stroke leading to significant morbidity and mortality [5]. While rare, limb loss has also been observed as a consequence of LVT requiring emergency surgical management [9]. In our case presented, our patient suffered acute MI and was found to have triple vessel disease through cardiac catheterization requiring CABG management. Shortly after recovery from his procedure, he had sudden onset of severe leg pain suggesting an acute embolic cause as opposed to in situ thrombosis, in which there would be a lack of severe pain due to the development of collateral circulation. Our patient subsequently underwent emergent surgical thromboembolectomy and was started on anticoagulation with significant improvement of his symptoms.

The management of LVT and associated embolization has been actively debated due to lack of randomized clinical control trials $[8,10]$. A cross-sectional study evaluating 35 patients with LV apical thrombi determined that the LVT resolved in all patients being treated with aspirin and warfarin. In five of the studied patients, LVT recurred after discontinuation of warfarin and therefore the patients required lifelong anticoagulation. Occurrence of LVT was linked to mostly akinesia or aneurysm with severe LV dysfunction. There were no reported thromboembolic events and the study concluded aspirin and warfarin therapy was appropriate for prophylaxis [11]. Systematic reviews, however, have determined no evidence for appropriate prophylaxis with anticoagulation in post-MI patients due to lack of benefit compared to hemorrhagic risk [12]. While warfarin use has been the mainstay of LVT anticoagulation, recent studies have evaluated the use of direct oral anticoagulants (DOACs) [13]. The ACC and American Heart Association (AHA) have concluded that DOACs can be considered as an alternative to warfarin therapy in patients with LVT post MI [5]. Due to the lack of clinical trials the discretion of which anticoagulation use remains up to the physician. In our patient presented, unfractionated heparin, oral clopidogrel and warfarin were used post-surgical thromboembolectomy, and he was discharged on continued warfarin, clopidogrel, and aspirin with no further thromboembolic events and decrease in LVT size on follow-up.

\section{Conclusions}

The incidence of LVT remains high in post-MI patients and complications of LVT are known to include thromboembolic events. Bilateral distal embolization due to LVT leading to critical limb ischemia remains a rare occurrence. Studies and trials focusing on the management and prevention of thromboembolic events have recommended the use of warfarin or DOACs, however, have determined to be inconclusive. In our presented case, the rapid diagnosis and emergent surgical treatment of limb-threatening embolism at the aortic bifurcation proved to have favorable outcomes for our patient. Furthermore, treatment with anticoagulation in our patient provided no further thromboembolic events and reduction in LVT size. Therefore, it is important for clinicians to recognize and promptly manage LVT and related arterial thromboembolic events. Further studies are also needed to develop an association between anticoagulation use and decreased morbidity and mortality in patients with LVT.

\section{Acknowledgments}

None to declare.

\section{Financial Disclosure}

The authors did not obtain funding for this manuscript.

\section{Conflict of Interest}

The authors declare no conflicts of interest for this publication.

\section{Informed Consent}

The patient described in the case report had given informed consent for the case report to be published.

\section{Author Contributions}

Khushboo K. Agarwal: case selection and drafting manuscript; 
Steven Douedi: discussion, drafting manuscript and revision; Abbas Alshami: drafting manuscript and revision; Brook DeJene: manuscript revision and final approval; Robert G. Kayser: planning, manuscript revision and final approval.

\section{Data Availability}

All data and information for this case report is presented in this manuscript.

\section{References}

1. Delewi R, Zijlstra F, Piek JJ. Left ventricular thrombus formation after acute myocardial infarction. Heart. 2012;98(23):1743-1749.

2. Habash F, Vallurupalli S. Challenges in management of left ventricular thrombus. Ther Adv Cardiovasc Dis. 2017;11(8):203-213.

3. Driesman A, Hyder O, Lang C, Stockwell P, Poppas A, Abbott JD. Incidence and predictors of left ventricular thrombus after primary percutaneous coronary intervention for anterior ST-segment elevation myocardial infarction. Clin Cardiol. 2015;38(10):590-597.

4. McCarthy CP, Murphy S, Venkateswaran RV, Singh A, Chang LL, Joice MG, Rivero JM, et al. Left Ventricular Thrombus: Contemporary Etiologies, Treatment Strategies, and Outcomes. J Am Coll Cardiol. 2019;73(15):2007-2009.

5. McCarthy CP, Vaduganathan M, McCarthy KJ, Januzzi JL, Jr., Bhatt DL, McEvoy JW. Left Ventricular Thrombus After Acute Myocardial Infarction: Screening, Prevention, and Treatment. JAMA Cardiol. 2018;3(7):642-649.
6. Hu HD, Chang Q, Chen Z, Liu C, Ren YY, Cai YC, Zhang J, et al. [Management and prognosis of acute arterial embolism: a multivariable analysis of 346 patients]. Zhonghua Yi Xue Za Zhi. 2011;91(41):2923-2926.

7. Stokman PJ, Nandra CS, Asinger RW. Left ventricular thrombus. Curr Treat Options Cardiovasc Med. 2001;3(6):515-521.

8. Shokr M, Ahmed A, Abubakar H, Sayedahmad Z, Rashed A, Afonso L, Cardozo S. Use of direct oral anticoagulants in the treatment of left ventricular thrombi: A tertiary center experience and review of the literature. Clin Case Rep. 2019;7(1):135-142.

9. Yeager RA, Moneta GL, Taylor LM, Jr., Hamre DW, McConnell DB, Porter JM. Surgical management of severe acute lower extremity ischemia. J Vasc Surg. 1992;15(2):385-391; discussion 392-383.

10. Phan J, Nguyen T, French J, Moses D, Schlaphoff G, Lo $\mathrm{S}$, Juergens $\mathrm{C}$, et al. Incidence and predictors of left ventricular thrombus formation following acute ST-segment elevation myocardial infarction: A serial cardiac MRI study. Int J Cardiol Heart Vasc. 2019;24:100395.

11. Ebrahimi M, Fazlinezhad A, Alvandi-Azari M, Abdar Esfahani M. Long-term clinical outcomes of the left ventricular thrombus in patients with ST elevation anterior myocardial infarction. ARYA Atheroscler. 2015;11(1):14.

12. Bastiany A, Grenier ME, Matteau A, Mansour S, Daneault B, Potter BJ. Prevention of left ventricular thrombus formation and systemic embolism after anterior myocardial infarction: a systematic literature review. Can J Cardiol. 2017;33(10):1229-1236.

13. Cochran JM, Jia X, Hamzeh I, et al. Direct oral anticoagulant use for left ventricular thrombus: a single center experience. Circulation. 2018;138:A16411. 